\title{
A SPLIT ACTION ASSOCIATED WITH A COMPACT TRANSFORMATION GROUP
}

\author{
SOL SCHWARTZMAN
}

\begin{abstract}
We associate with an effective action of a compact connected Lie group as a pathwise connected space $X$ a split action of a quotient group $G / K$ on the quotient space $X / K$. One application of the main theorem states that if $X$ is a compact oriented manifold whose principal cohomology class is a cup product of one-dimensional classes then the action of $G$ on $X$ splits. We prove this in the differentiable case; the topological case has since been dealt with by Schultz.
\end{abstract}

The results of this paper are closely related to those in Injective operations of the toral groups by Conner and Raymond [3]. In what follows $G$ will always be a compact connected topological group and $X$ will be a pathwise connected Hausdorff space on which $G$ acts. We will say that an action of $G$ splits provided it is equivariantly isomorphic to an action of $G$ on a product space $Y \times H$, where $G$ acts trivially on the first factor $Y$ and transitively on the second factor $H$. If $G$ is commutative then an effective split action of $G$ is simply a principal bundle action where the bundle is a trivial (i.e., product) bundle.

We are going to associate with our transformation group $G$ a canonically defined subgroup $K$ such that the induced action of $G$ on $X / K$ splits. It will turn out that $K$ is normal and $G / K$ is commutative. Once we have proved this we will get new proofs of the splitting and fibering theorems in [3]. We will also get an application to the case where $G$ is a Lie group acting differentiably on a compact oriented manifold $X$. In this situation, if we assume that the fundamental cohomology class of $X$ is a cup product of one-dimensional classes, it will turn out that our subgroup $K$ consists of the identity element, so the action splits. Other applications will be given below. After learning of this result Schultz proved a topological version of this theorem as well as a strengthened version of Theorem 5 (Schultz [6]).

Central to our discussion will be a consideration of continuous functions from a topological space into the multiplicative group $T^{1}$ of complex numbers of absolute value one. If $X$ is a topological space, let $C(X)$ denote the set of all such functions, made into a group under pointwise multiplication. Let $R(X)$ be the subgroup of $C(X)$ consisting of all functions $f(x)$ in $C(X)$ for which there exists a continuous real-valued function $h(X)$ such that $f(x)=\exp 2 \pi i h(x)$. Then $R(X)$ consists of all functions in $C(X)$ which are homotopic to a constant map. We associate with each space $X$ the group $C(X) / R(X)$ and in this way get a contravariant functor from

Received by the editors July 16, 1979 and, in revised form, October 4, 1980.

1980 Mathematics Subject Classification. Primary 54H15, 57S10.

Key words and phrases. Equivariant map, split action.

() 1981 American Mathematical Society 0002-9939/81/0000-0580/\$03.00 
the category of topological spaces and continuous maps into the category of commutative groups and homomorphisms. If we restrict this functor to the category of arcwise connected spaces of the homotopy type of a $\mathrm{CW}$ complex, it is a well-known fact that the resulting functor is naturally equivalent to the one-dimensional singular cohomology functor. For the purposes of this paper it will be convenient to denote $C(X) / R(X)$ by $H^{\prime}(X)$ for all spaces $X$. This is a torsion-free group. If $G$ is a compact connected topological group the collection $\Lambda(G)$ of continuous homomorphisms of $G$ into $T^{\prime}$ is a group under pointwise multiplication. Each equivalence class in $C(G) / R(G)$ contains exactly one element of $\Lambda(G)$, so $\Lambda(G)$ is isomorphic to $H^{1}(G)$.

We return now to our standing assumption that $X$ is a pathwise connected Hausdorff space and $G$ is a compact connected group acting on $X$. Since we are assuming that $X$ is pathwise connected, if we let $f_{x}^{*}: G \rightarrow X$ be defined by $f_{x}^{*}(g)=g x$, the homotopy class of $f_{x}^{*}$ is independent of $x$. We thus get a uniquely defined homomorphism of $H^{1}(X)$ into $H^{1}(G)$, and by virtue of the isomorphism of $H^{1}(G)$ with $\Lambda(G)$ we have a homomorphism $h$ of $H^{\prime}(X)$ into $\Lambda(G)$. If either $G$ is a Lie group or $H^{1}(X)$ is finitely generated, the image of $h$ is a finitely generated free abelian group, since $H^{1}(G)$ is torsion free.

Now let $K$ be the subgroup of $G$ which is the intersection of all the kernels of the homomorphisms of $G$ into $T^{1}$ which lie in the image of $h$. Obviously $K$ is a normal subgroup of $G$, and since the homomorphisms of $G / K$ into the commutative group $T^{1}$ distinguish between points of $G / K$, it follows that $G / K$ is commutative.

If we let $X / K$ be the orbit space of $X$ under the action of $K$, there is a natural action of $G$ on $X / K$, and the projection map of the $G$-space $X$ onto the $G$-space $X / K$ is equivariant.

THEOREM 1. If either $G$ is a Lie group or $H^{1}(X)$ is finitely generated, the action of $G$ on $X / K$ splits and is equivariantly isomorphic to the obvious split action of $G$ on $X / G \times G / K$. Moreover the map of $H^{1}(X / K)$ into $H^{1}(X)$ induced by projection is an isomorphism. Finally if $G$ is commutative, any equivariant map of $X$ into a split action of $G$ can be factored equivariantly through the projection of $X$ onto $X / K$.

It is perhaps worth noting that if $G$ is a semisimple Lie group it follows from the fact that $G / K$ is commutative that $G=K$. Thus in this case we can conclude that the map of $H^{1}(X / G)$ into $H^{1}(X)$ induced by projection is an isomorphism.

Before proceeding to the proof of our main theorem we will need the following

Definition. An eigenfunction for the action of $G$ on $X$ is an element $f(x) \in$ $C(X)$ for which there exists a $\chi(g) \in \Lambda(G)$ such that for all $x \in X$ and $g \in G$, $f(g x)=\chi(g) f(x)$.

Note that if $f$ is an eigenfunction, the associated $\chi(g)$ must be the image under $h$ of the element of $C(X) / R(X)$ determined by $f$.

Now let $f(x) \in C(X)$ and let $[f]$ be the element of $H^{1}(X)=C(X) / R(X)$ determined by $f$. Let $\chi(g)$ be the element of $\Lambda(G)$ which is the image of $[f]$ under $h$. Since $[\chi(g)]^{-1}=\chi\left(g^{-1}\right), f(g x) \chi\left(g^{-1}\right)$ can be written in the form $\exp (2 \pi i \lambda(x, g))$ where $\lambda(x, g)$ is a real valued function which is continuous in $g$ for each fixed 
$x \in X$. If $\lambda_{1}(x, g)$ and $\lambda_{2}(x, g)$ are two such functions their difference must be an integer valued function which depends on $x$ alone, since it is continuous in $g$ and $G$ is connected. Thus if we let $\mu$ be Haar measure on $G$ and define

$$
\bar{f}(x)=\exp 2 \pi i \int \lambda(x, g) d \mu(g),
$$

$\bar{f}(x)$ is independent of the particular $\lambda(x, g)$ we use

LEMMA 1.1. $\bar{f}(x) \in C(X)$ and $f$ is homotopic to $\bar{f}$. Moreover, for all $x \in X$ and $g \in G, \bar{f}(g x)=\chi(g) \bar{f}(x)$.

In other words we are going to show that each equivalence class in $C(X) / R(X)$ contains an eigenfunction.

Proof. We first show that $\bar{f}(x)$ is continuous. Denote the multiplicative group of complex numbers of absolute value one by $T^{1}$. Then, using the usual notation for function spaces, $f(x, g) \chi\left(g^{-1}\right) \in\left(T^{1}\right)^{G \times X}$, which is homeomorphic to $\left[\left(T^{1}\right)^{G}\right]^{X}$. Thus if $x_{0} \in X$, there is an open set $O$ containing $x_{0}$ such that for $x$ in $O$ and any $g \in G,\left|f(g x) \chi\left(g^{-1}\right)-f\left(g x_{0}\right) \chi\left(g^{-1}\right)\right|<\frac{1}{2}$. This implies that on $O \times G, f(g x) \chi\left(g^{-1}\right)$ is homotopic to $f\left(g x_{0}\right) \chi\left(g^{-1}\right)$ which is independent of $x$ and has a continuous logarithm (i.e., is homotopic to a constant map). Thus the restriction of $f(x g) x\left(g^{-1}\right)$ to $O \times G$ has a continuous logarithm, i.e., $\lambda(x, g)$ can be chosen to be continuous on $O \times G$. Denoting the additive group of the real line by $R^{1}$, the restriction of such a $\lambda(x, g)$ to $O \times G$ is an element of $\left(R^{1}\right)^{O \times G}$ which is homeomorphic to $\left(\left(R^{1}\right)^{G}\right)^{O}$. Thus, given any $\varepsilon>0$ we can find an open set $O^{1}$ such that $x_{0} \in O^{1} \subseteq$ $O$ and for $x \in O$ and $g \in G,\left|\lambda(x, g)-\lambda\left(x_{0}, g\right)\right|<\varepsilon$. From this it follows that $\bar{f}(x)$ is continuous.

To see that $\bar{f}(x)$ and $f(x)$ are homotopic maps into $T^{1}$, let $\mu_{e}$ be the unit measure on the Borel sets of $G$ which is concentrated at the identity. Define $H(x, t)$ to equal

$$
\exp \left(2 \pi i \int \lambda(x, g) d\left(t u(g)+(1-t) \mu_{e}(g)\right)\right) \text {. }
$$

Then $H(x, t)$ is continuous and $H(x, 0)=f(x)$ while $H(x, 1)=\bar{f}(x)$.

It remains to prove that $\bar{f}(x)$ is an eigenfunction. For any fixed $g_{0} \in G$, let $\alpha_{0}$ be a real number such that $\chi\left(g_{0}\right)=\exp 2 \pi i \alpha_{0}$. Then for any $x \in X$ and $g \in G$,

$$
\begin{aligned}
\exp 2 \pi i \lambda\left(g_{0} x, g\right) & =f\left(g g_{0} x\right) \chi\left(g^{-1}\right) \\
& =f\left(g g_{0} x\right) \chi\left(\left(g g_{0}\right)^{-1}\right) \chi\left(g_{0}\right)=\exp 2 \pi i\left(\alpha_{0}+\lambda\left(x, g g_{0}\right)\right) .
\end{aligned}
$$

Thus $\lambda\left(g_{0} x, g\right)$ differs from $\alpha_{0}+\lambda\left(x, g g_{0}\right)$ by an integer valued function which by continuity in $g$ depends only on $x$ (since $G$ is connected). But then

$$
\begin{aligned}
\bar{f}\left(g_{0} x\right) & =\exp 2 \pi i \int \lambda\left(g_{0} x, g\right) d \mu(g)=\exp 2 \pi i \int \alpha_{0}+\lambda\left(x, g g_{0}\right) d \mu(g) \\
& =\chi\left(g_{0}\right) \exp 2 \pi i \int \lambda(x, g) d \mu(g)=\chi\left(g_{0}\right) \bar{f}(x) .
\end{aligned}
$$

This completes the proof of our lemma. To prove the main theorem, we begin by recalling that the assumption that either $G$ is a Lie group or $H^{1}(X)$ is finitely generated assures us that the image of $H^{1}(X)$ in $\Lambda(G)$ under $h$ is a finitely 
generated free commutative group. Therefore by our lemma we can pick eigenfunctions $f_{1}, \ldots, f_{k}$ such that $h\left[f_{1}\right], \ldots, h\left[f_{k}\right]$ is a basis for this image. Let $h\left[f_{i}\right]$ be denoted by $\chi_{i}$. Then $f_{i}(g x)=\chi_{i}(g) f_{i}(x)$ for all $g \in G$ and $x \in X$.

Let $\sigma: G \rightarrow T^{k}$ be defined by $\sigma(g)=\left(\chi_{1}(g), \ldots, \chi_{k}(g)\right)$. Then $\sigma$ is a homomorphism of $G$ into the multiplicative group $T^{k}$ of $k$-tuplets of complex numbers of absolute value one. The kernel of $\sigma$ is obviously $K$.

LEMMA 1.2. $\sigma$ is surjective.

Proof. The image of $G$ under $\sigma$ is a closed subgroup of $T^{k}$. If it is a proper subgroup, we can get a nontrivial element of $\Lambda\left(T^{k}\right)$ which is equal to one on this image. This can be seen by noting that the quotient of $T^{k}$ by this proper subgroup would be a toral group. Recall now that a nontrivial element of $\Lambda\left(T^{k}\right)$ is of the type $\rho\left(z_{1}, \ldots, z_{k}\right)=z_{1}^{n_{1}} \cdots z_{k}^{n_{k}}$ where not all the $n_{i}$ are zero. Thus if $\sigma$ is not surjective there would exist integers $n_{1}, \ldots, n_{k}$ not all zero such that $\chi_{1}^{n_{1}} \cdots \chi_{k}^{n_{k}}$ $=1$. This however would contradict the fact that $\chi_{1}, \ldots, \chi_{k}$ is a basis for the image of $h$. This completes the proof of the lemma.

We make $T^{k}$ into a $G$-space by defining $g\left(z_{1}, \ldots, z_{k}\right)=\left(\chi_{1}(g) z_{1}, \ldots, \chi_{k}(g) z_{k}\right)$. By our lemma this is a transitive action.

Let $T: X \rightarrow X / G$ be the projection and define $P: X \rightarrow X / G \times T^{k}$ by $P(x)=$ $\left(T(x), f_{1}(x), \ldots, f_{k}(x)\right)$. We make $X / G \times T^{k}$ into a $G$-space by defining $g\left(\bar{x}, z_{1}, \ldots, z_{k}\right)=\left(\bar{x}, \chi_{1}(g) z_{1}, \ldots, \chi_{k}(g) z_{k}\right)$; this is obviously a split action, and $P$ is equivariant. From the fact that $\sigma$ is surjective and has kernel $K$ it follows that $T^{k}$ can be identified with $G / K$ and the split action of $G$ on $X / G \times T^{k}$ is equivariantly isomorphic to the obvious split action of $G$ on $X / G \times G / K$. Note also that $P$ is surjective.

The map $P$ can be factored equivariantly through the projection of $X$ onto the $G$-space $X / K$. From the fact that $\sigma$ is surjective and has kernel $K$ it follows that we get in this way a one-one equivariant map of the $G$-space $X / K$ onto the $G$-space $X / G \times T^{k}$. To see that this is a homeomorphism we need only show that $P$ is open.

To see that $P$ is open, let $x_{0} \in X$ and suppose $O$ is an open set containing $x_{0}$. There is an open set $O^{\prime}$ in $X$ and an open set $U$ in $G$ containing the identity such that $X_{0} \in O^{\prime} \subseteq O$ and for $g \in U$ and $y \in O^{\prime}, g y \in O$. We can then choose an open set $V$ in $G$ containing the identity such that $V=V^{-1}, V V \leqslant U$.

Now let $\lambda: X \rightarrow T^{k}$ be defined by $\lambda(x)=\left(f_{1}(x), \ldots, f_{k}(x)\right)$. Then we can pick $O^{\prime \prime} \leqslant X$ such that $O^{\prime \prime}$ is open, $x_{0} \in O^{\prime \prime} \leqslant O^{\prime} \leqslant O$ and for any $y$ in $O^{\prime \prime}$, $\lambda(y) / \lambda\left(x_{0}\right)$ belongs to the image of $V$ under $\sigma$. Then for any $y \in O^{\prime \prime}$ we can pick $g_{y} \in V$ such that $\lambda\left(g_{y} y\right)=\lambda\left(x_{0}\right)$. From this it follows that $P(O)$ contains $\left(T(y), z_{1}, \ldots, z_{k}\right)$ for any $y \in O^{\prime \prime}$ and any $\left(z_{1}, \ldots, z_{k}\right)$ in the translate of $\lambda\left(x_{0}\right)$ by $\sigma(V)$. Thus $P\left(x_{0}\right)$ is an interior point of $P(O)$, so $P$ is open.

We have thus shown that the action of $G$ on $X / K$ splits and is equivariantly isomorphic to the obvious action of $G$ on $X / G \times G / K$, both of these being equivariantly isomorphic to the action we have defined on $X / G \times T^{k}$. We wish next to show that the map of $H^{1}(X / K)$ into $H^{1}(X)$ induced by projection is 
surjective. By virtue of our equivariant isomorphism of $X / K$ with $X / G \times T^{k}$ we need only show that $H^{1}(P)$ is surjective. Let $f \in C(X)$. If $\chi=h([f])$ there is an eigenfunction $\bar{f} \in C(X)$ such that $[\bar{f}]=[f]$ and $\bar{f}(g x)=\chi(g) \bar{f}(x)$. But there exist integers $n_{1}, \ldots, n_{k}$ such that $\chi=\chi_{1}^{n_{1}}, \ldots, \chi_{k}^{n_{k}}$. If we let $F=\bar{f} / f_{1}^{n_{1}}, \ldots, f_{k}^{n_{k}}$, then $F(g x)=F(x)$ identically so $F$ arises from a function on $X / G$. Thus $f$ arises from a function on $X / G \times T^{k}$, so $[f]=[\bar{f}]$ lies in the image of $H^{1}(P)$. Thus $H^{1}(P)$ is surjective. Note that this argument shows that any eigenfunction on $X$ arises from one on $X / G \times T^{k}$.

To see that the map of $H^{1}(X / K)$ into $H^{1}(X)$ is injective we use an indirect proof. If the map is not injective there exists a function $\alpha \in R(X)$ such that $\exp 2 \pi i \alpha(k x)=\exp 2 \pi i \alpha(x)$ for all $k \in K$ and $x \in X$ while the element of $C(X / K)$ determined by $\exp 2 \pi i \alpha(x)$ does not yield the identity element of $H^{1}(X / K)$. But for any $k_{0} \in K, \alpha\left(k_{0} x\right)-\alpha(x)$ is a continuous integer valued function on the connected space $X$ and is therefore equal to a constant $n_{0}$. But then $\alpha\left(k_{0}^{r} x\right)-\alpha(x)$ clearly equals $r n_{0}$. Since the orbit of any $x \in X$ under the action of $K$ is compact, $\alpha$ must be bounded on this orbit so $n_{0}=0$. Thus for all $k \in K$ and $x \in X, \alpha(k x)=\alpha(x)$ which contradicts our assumptions about $\alpha$.

Finally suppose that $G$ is commutative and suppose that $Q$ is an equivariant map of $X$ onto $Y \times G / K^{1}$ where $G$ acts trivially on $Y$ and in the obvious way on $G / K^{1}$. If $q_{1}$ and $q_{2}$ are the projections of $Y \times G / K^{1}$ on $Y$ and $G / K^{1}$ respectively, it is clear that $q_{1} \circ Q$ can be factored through the projection of $X$ on $X / K$. The duality theory for locally compact commutative groups tells us that the functions in $\Lambda\left(G / K^{1}\right)$ distinguish between points of $G / K^{1}$. To show that $q_{1} \circ Q$ can be factored through the projection of $X$ on $X / K$ it is therefore only necessary to show that for each $\chi^{1} \in \Lambda\left(G / K^{1}\right), \chi^{1} \circ q_{2} \circ Q$ can be factored through the projection of $X$ on $X / K$. However

$$
\begin{aligned}
\left(\chi^{1} \circ q_{2} \circ Q\right)(g x) & =\chi^{1}\left((\lambda(g))\left(q_{2} \circ Q\right)(x)\right) \\
& =\chi^{1}(\lambda(g))\left(\left(\chi^{1} \circ q_{2} \circ Q\right)(x)\right)
\end{aligned}
$$

where $\lambda$ is the projection of $G$ on $G / K^{1}$. Since $\chi^{1} \circ \lambda$ is a homomorphism of $G$ into $T^{1}, \chi^{1} \circ q_{2} \circ Q$ is an eigenfunction on $X$. To complete the proof of our theorem then we need only see that every eigenfunction on $X$ can be factored through the projection of $X$ on $X / K$. By virtue of the equivariant isomorphism of $X / K$ with $X / G \times T^{k}$ it is enough to see that every eigenfunction can be factored through the projection $P$ we defined of $X$ on $X / G \times T^{k}$. However in our proof that $H^{1}(P)$ is surjective we establish this.

Now if $G$ is effective and commutative, by what we have shown the action of $G$ splits if and only if $K$ consists of the identity element. By the duality theory for locally compact groups this happens if and only if the image of the homomorphism $h$ of $H^{1}(X)$ into $\Lambda(G)$ is all of $\Lambda(G)$. Thus we get the following

THEOREM 2. If $G$ is effective and commutative and either $G$ is a Lie group or $H^{1}(X)$ is finitely generated, the action of $G$ on $X$ splits if and only if the obvious map of $H^{1}(X)$ into $H^{1}(G)$ is surjective. 
Now suppose $X$ has the homotopy type of a CW complex, so that we can identify $H^{1}(X)$ with the one-dimensional singular cohomology group of $X$. Assume further in the case that $G$ is not a toral group that $G$ is arcwise connected. Then we obtain the following result. (The maps of groups referred to are those induced by a map $f_{x}: G \rightarrow X$ defined by $f_{x}(g)=g x$. The homotopy class of $f_{x}$ is of course independent of $x$.)

COROLlARY. Assume either $G$ is a toral group or $H^{1}(X)$ is finitely generated and $G$ is commutative, and that $G$ acts effectively. Then the following are equivalent:

(a) The action of $G$ on $X$ splits.

(b) $\pi_{1}(G, e)$ is mapped injectively onto a direct summand in $\pi_{1}\left(X, x_{0}\right)$.

(c) $H_{1}(G)$ is mapped injectively onto a direct summand in $H_{1}(X)$.

(d) $H^{1}(X)$ is mapped surjectively onto $H^{1}(G)$.

The fact that (a) $\rightarrow$ (b), (b) $\rightarrow$ (c), and (c) $\rightarrow$ (d) is trivial and we have already shown that $(d) \rightarrow(a)$.

The equivalence of (a) and (b) is implicit in the splitting theorem proved in [3]. To complete the connection between our discussion and that in [3], assume $X$ is semilocally simply connected. Then with any subgroup $H$ of $\pi_{1}\left(X, x_{0}\right)$ we associate a covering space of $X$. It is well known that the action of $G$ on $X$ can be lifted to this covering space if and only if the image of $\pi_{1}(G, e)$ in $\pi_{1}\left(X, x_{0}\right)$ is contained in $H$. Then by our corollary this lifted action splits if and only if $\pi_{1}(G, e)$ is mapped injectively onto a direct summand in $H$.

For simplicity now, assume that $G$ is a toral group acting effectively. For any closed subgroup $L$ of $G$, there is an obvious action of $G$ on $G / L$. Trivially this is a split action.

Definition. The $G$-space $X$ fibres equivariantly over $G / L$ provided there is an equivariant map of $X$ onto $G / L$. (Note that such a map must factor through the projection of $X$ on $X / K$.)

It can be seen that such an equivariant map is in fact a locally trivial fibration with structure group $L$. It is now obvious that $X$ fibres equivariantly over $G / L$ if and only if $L \supseteq K$. Now the dimension of $K$ equals the dimension of $G$ minus the rank of the image in $\Lambda(G)$ of our homomorphism $h$ of $H^{1}(X)$ into $\Lambda(G)$. Thus we get

THEOREM 3. $X$ fibres equivariantly over some $G / L$ with $L$ finite if and only if the rank of the image of the usual map of $H^{1}(X)$ in $H^{1}(G)$ equals the dimension of $G$. More generally $X$ fibres equivariantly over some $G / L$ with the dimension of $L=k$ if and only if the image of the usual map of $H^{1}(X)$ into $H^{1}(G)$ has $\operatorname{ran} K \geqslant n-k$.

These results are implicit in [4].

It follows from what we have done that if $X$ does not fibre over a circle then $K=G$ and therefore the obvious map of $H^{1}(X / G)$ into $H^{1}(X)$ is an isomorphism.

Next we consider the situation in which $G$ is a compact connected Lie group acting differentiably and effectively on a compact oriented differentiable manifold $X$. We will again denote by $K$ the subgroup of $G$ which arises in our main theorem. 
THEOREM 4. If the fundamental cohomology class is a cup product of one-dimensional classes (integer coefficients) then $K$ consists of just the identity element, so $G$ is a toral group and the action of $G$ on $X$ splits.

If instead some nonzero multiple of the fundamental cohomology class is a cup product of one-dimensional classes then it will be clear from our proof that $K$ is finite, so in this case we can conclude that $G$ is a toral group. This latter fact was already known. We should also note that in the case in which $X$ is a torus the fact that the action splits was already known [1].

To prove the theorem we use the fact that $H^{1}(X / K)$ maps surjectively onto $H^{1}(X)$. Let $\lambda_{1}, \ldots, \lambda_{r}$ be elements of $H^{1}(X)$ whose cup product is the fundamental cohomology class of $X$, and let $\mu_{1}, \ldots, \mu_{r}$ be elements of $H^{\prime}(X / K)$ which map into $\lambda_{1}, \ldots, \lambda_{r}$. Then obviously $\mu_{1} \cup \cdots \cup \mu_{r}$ maps into the fundamental cohomology class of $X$. Since $H^{r}(X / K)$ is nontrivial the dimension of $X / K$ must equal $r$, the dimension of $X$. Therefore $K$ must be finite. For some prime $p$ we can pick an injection $i$ of $Z_{p}$ into $K$, if $K$ is nontrivial. Because $G$ is arcwise connected the elements of $i\left(Z_{p}\right)$ determine homeomorphisms on $X$ which are isotopic to the identity map. Therefore the induced action of $i\left(Z_{p}\right)$ on $X$ is orientation preserving. If $K$ is nontrivial then it is clear that we get an effective differentiable orientation preserving action of $Z_{p}$ on $X$ such that $H^{r}\left(X / Z_{p}\right)$ maps surjectively on $H^{r}(X)$, where $r$ is the dimension of $X$. I am indebted to Professor R. Z. Goldstein of SUNY at Albany for the proof that this cannot occur. His proof follows.

Let $F$ be the fixed point set of $Z_{p}$. Then $F$ is a manifold of dimsension less than or equal to $r-2$. Let $U$ be an invariant open tubular neighborhood of $F$ and let $B$ be its boundary. Consider the following commutative diagram.

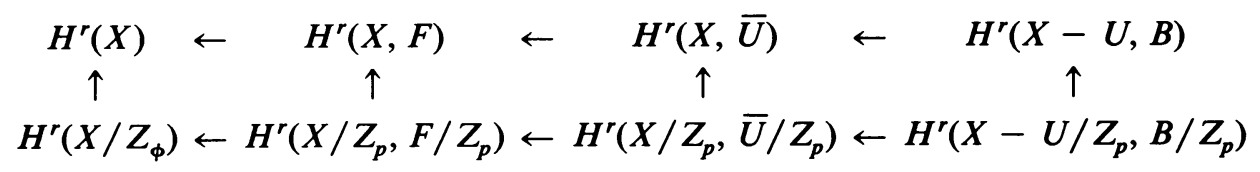

Clearly the horizontal maps are all isomorphisms. However the map of $X-U$ onto $X-U / Z_{p}$ is a $p$ to one covering map. Since $X-U / Z_{p}$ is a differentiable manifold we can triangulate it. This triangulation lifts to $X-U$. If we use oriented simplicial cohomology it is clear that the generator of $H^{r}\left(X-U / Z_{p}, B / Z_{p}\right)$ is sent onto $p$ times the generator of $H^{r}(X-U, B)$, and therefore the same is true of the map of $H^{r}\left(X / Z_{p}\right)$ into $H^{r}(X)$. This completes the proof.

Finally let us again assume that we have a compact oriented differentiable manifold $X$ such that the fundamental cohomology class is a cup product of one-dimensional classes. Suppose further that $\phi$ is a differentiable homeomorphism of $X$ onto itself of period exactly $n$.

THEOREM 5. If $\phi$ is isotopic to the identity map, $\phi$ acts freely, i.e., $\phi$ generates a free action of $Z_{n}$ on $X$.

Proof. We form $X \times{ }_{z_{n}} T^{1}$ using our $Z_{n}$ action on $X$ and consider the usual action of $T^{1}$ on $X \times_{Z_{n}} T^{1}$. Because $\phi$ is isotopic to the identity, $X \times_{Z_{n}} T^{1}$ is 
homeomorphic to $X \times T^{1}$. Since the fundamental cohomology class of $X \times T^{1}$ is the cup product of the liftings via the projection maps of the fundamental cohomology classes of $X$ and $T^{1}$, the assumptions of the previous theorem hold and the action of $T^{1}$ on $X \times{ }_{Z_{n}} T^{1}$ splits.

Thus in particular the action of $T^{1}$ on $X \times{ }_{Z_{n}} T^{1}$ is a principal bundle action. However this implies that $Z_{n}$ acts freely on $X$.

\section{REFERENCES}

1. P. E. Conner and D. Montgomery, Transformation groups on a $K(\pi, 1)$, Michigan Math. J. 6 (1959), 405-412.

2. P. E. Conner and Frank Raymond, Actions of compact Lie groups on aspherical manifolds, Topology of Manifolds, Markham, Chicago, Ill., 1969, pp. 222-264.

3. __ Injective operations of the toral groups, Topology 11 (1971), 283-296.

4. Injective operations of the toral groups. II (Proc. Second Conf. on Compact Transformation Groups, Univ. of Massachusetts, Amherst, 1971), Lecture Notes in Math., vol. 299, Springer, Berlin and New York, 1972, pp. 109-123.

5. Dan Burghelea and Reinhard Schultz, On the semi-simple degree of symmetry, Bull. Soc. Math. France 103 (1975), 443-440.

6. Reinhard Schultz, Group actions on hypertoral manifolds. I, II (to appear).

Department of Mathematics, University of Rhode Island, Kingston, Rhode IsLand 02881 